\title{
New observational constraints on $f(T)$ gravity through gravitational-wave astronomy
}

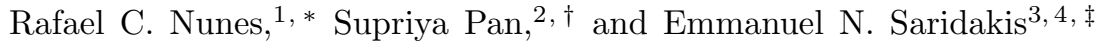 \\ ${ }^{1}$ Divisão de Astrofísica, Instituto Nacional de Pesquisas Espaciais, \\ Avenida dos Astronautas 1758, São José dos Campos, 12227-010, SP, Brazil \\ ${ }^{2}$ Department of Mathematics, Presidency University, 86/1 College Street, Kolkata 700073, India \\ ${ }^{3}$ Department of Physics, National Technical University of Athens, Zografou Campus GR 157 73, Athens, Greece \\ ${ }^{4}$ CASPER, Physics Department, Baylor University, Waco, TX 76798-7310, USA
}

\begin{abstract}
We investigate the new observational constraints on $f(T)$ gravity that arise from the effects of primordial gravitational waves $(\mathrm{GWs})$ on the cosmic microwave background (CMB) anisotropies and the BB spectrum. We first show that on the GWs propagation in $f(T)$ gravity we obtain only an amplitude modification and not a phase one, comparing to the case of general relativity in the background of $\Lambda \mathrm{CDM}$ cosmology. Concerning primordial GWs we find that the more the model departs from general relativity the larger is the GW amplitude decay, and thus a possible future detection would bring the viable $f(T)$ gravity models five orders of magnitude closer to $\Lambda$ CDM cosmology comparing to standard cosmological constraints. Additionally, we use the CLASS code and both data from the Planck probe, as well as forecasts from the near-future CORE collaboration, and we show that possible non-trivial constraints on the tensor-to-scalar ratio would offer a clear signature of $f(T)$ gravity. Finally, we discuss on the possibility to use the properties of the GWs that arise from neutron stars mergers in order to extract additional constrains on the theory.
\end{abstract}

PACS numbers: 98.80.-k, 95.36.+x, 04.50.Kd, 04.30.Nk

\section{INTRODUCTION}

The LIGO and Virgo collaborations reported the first direct detection of gravitational waves (GWs) through the GW150914 event [1, that was produced from the merger of a pair of black holes with masses around 36 and 29 solar masses and the subsequent ringdown of a single black hole, while similar GWs event were later reported in 2 [6. Recently, the detection of a binary neutron star merger with a GW (GW170817 event [7) and an electromagnetic counterpart (GRB 170817A event 8]) opened the window of multi-messenger GW astronomy. Certainly, the detection of GWs is a potential indication for a new era in modern astrophysics and cosmology, offering a new spectrum of possibilities to investigate nature at the fundamental level.

On the other hand, another important aspect in cosmology is to investigate the cosmic origin through a possible detection of primordial gravitational waves and their effect on the Cosmic Microwave Background (CMB) in the very low frequency band, which is expected to bring new astrophysical and cosmological information. The primordial GWs can be quantified through the tensor-toscalar ratio $r$ evaluated on some pivot scale. The Planck collaboration within $\Lambda \mathrm{CDM}+r$ has reported $r<0.10$ at $95 \%$ confidence level (CL) by combining temperature, low-polarization, and lensing, at the pivot scale $k_{*}=0.002 \mathrm{Mpc}^{-1}[9]$.

Concerning the research on the fundamental gravita-

\footnotetext{
*Electronic address: rafadcnunes@gmail.com

$\dagger$ Electronic address: supriya.maths@presiuniv.ac.in

‡Electronic address: Emmanuel_Saridakis@baylor.edu
}

tional interaction, the GWs detection can be extremely helpful since the underlying gravitational theory determines both the properties of the GWs themselves (speed, polarization modes etc) as well as the properties of the background on which they propagate (the expanding universe). Thus, one can use the GWs measured properties and extract valuable information for the structure of the fundamental gravitational interaction. For instance, from the recent GW170817 and GRB 170817A events, which showed that GWs propagate practically with the light speed, one may impose strong constraints or exclude various modified gravity theories of the literature [10 13. (see 14 for a latest review). Implications of an electromagnetic counterpart measurement for a range of modified gravity theories was also previously discussed in 15, 16.

One class of theories of modified gravity that have recently attracted the interest of the research is the torsional gravitational modification, such as the $f(T)$ gravity. In this theory one describes the gravitational interaction through the torsion instead of the curvature tensor, and thus the Lagrangian is a function of the torsion scalar $T$ (see [17] for a review). Hence, $f(T)$ gravity corresponds to a novel gravitational modification, with no known curvature equivalence, and it proves to lead to interesting cosmology at both early and late times 18 50. and be in agreement with observations 51 56.

Some preliminary investigations concerning the GWs properties in $f(T)$ gravity have been performed in [57, 58, where it was shown that their speed is equal to the light speed and thus the constraints from GW170817 are trivially satisfied (see also [59, 60] for the GWs properties in other modified teleparallel gravities). In this work we are interested in studying the new observational constraints that can be imposed on $f(T)$ gravity from the 
advancing multi-messenger GW astronomy. Moreover, we examine the effect which primordial GWs have on the cosmic microwave background (CMB) anisotropies and we examine the corresponding constraints. Finally, we discuss on the possibility that GWs from neutron star mergers could lead to additional constraints on the theory.

The manuscript is organized as follows: In Section II we present the equations that determine the propagation of GWs in $f(T)$ gravity, and we quantify their modification comparing to the case of general relativity in a background of $\Lambda$ CDM cosmology. In Section III we study the observational constrains that arise from primordial GWs, and in Section IV we use the CLASS code and data from the Planck probe and forecasts from the near future CORE collaboration in order to quantify the effects of primordial GWs on the CMB anisotropies and the BB spectrum. In Section $\mathrm{V}$ we discuss on the possibility to extract constraints from GWs that arise from neutron star mergers. Finally, in Section VI we present the conclusions.

\section{GRAVITATIONAL WAVES IN $f(T)$ GRAVITY}

In this section we describe the equations of $f(T)$ gravity, as well as the formulation of the tensor modes propagation in this cosmological context.

In the framework of torsional gravity ones uses the tetrad fields $e_{A}^{\mu}$, which form an orthonormal base in the tangent space of the underlying manifold $\left(\mathcal{M}, g_{\mu \nu}\right)$, where $g_{\mu \nu}=\eta_{A B} e_{\mu}^{A} e_{\nu}^{B}$ is the metric tensor defined on the manifold $\mathcal{M}$. Throughout this work we use the Greek indices to denote the coordinate space and the Latin indices for the tangent space. Furthermore, unlike general relativity which uses the torsionless Levi-Civita connection, here we use the curvatureless Weitzenböck connection $\stackrel{\mathrm{w}}{\Gamma}_{\nu \mu}^{\lambda} \equiv e_{A}^{\lambda} \partial_{\mu} e_{\nu}^{A}[61$. Thus, the gravitational field is described by the torsion tensor

$$
T_{\mu \nu}^{\lambda} \equiv e_{A}^{\rho}\left(\partial_{\mu} e_{\nu}^{A}-\partial_{\nu} e_{\mu}^{A}\right)
$$

The Lagrangian of the teleparallel equivalent of general relativity (TEGR) is the torsion scalar $T$, constructed as 61

$$
T \equiv \frac{1}{4} T^{\rho \mu \nu} T_{\rho \mu \nu}+\frac{1}{2} T^{\rho \mu \nu} T_{\nu \mu \rho}-T_{\rho \mu}{ }^{\rho} T_{\nu}^{\nu \mu},
$$

and the corresponding action reads $S=\frac{1}{16 \pi G} \int d^{4} x e T$, where $e=\operatorname{det}\left(e_{\mu}^{A}\right)=\sqrt{-g}$ and $G$ is the Newton's gravitational constant (we set the speed of light to $c=1$ ).

If we use TEGR as the starting point for torsional modified gravity, the simplest such modification is $f(T)$ gravity, with action

$$
S=\frac{1}{16 \pi G} \int d^{4} x e f(T)
$$

Variation of (3) with respect to the tetrads leads to the field equations

$$
\begin{aligned}
& e^{-1} \partial_{\mu}\left(e e_{A}^{\rho} S_{\rho}{ }^{\mu \nu}\right) f_{T}+e_{A}^{\rho} S_{\rho}{ }^{\mu \nu} \partial_{\mu}(T) f_{T T} \\
& -f_{T} e_{A}^{\lambda} T_{\mu \lambda}^{\rho} S_{\rho}{ }^{\nu \mu}+\frac{1}{4} e_{A}^{\nu} f(T)=4 \pi G e_{A}^{\rho} \Theta_{\rho}{ }^{\nu},
\end{aligned}
$$

where $f_{T}=\partial f / \partial T, f_{T T}=\partial^{2} f / \partial T^{2}$, and $\Theta_{\rho}{ }^{\nu}$ denotes the energy-momentum tensor of the matter sector. In the above equation we have introduced for convenience the "super-potential" $S_{\rho}^{\mu \nu} \equiv \frac{1}{2}\left(\mathcal{K}_{\rho}^{\mu \nu}+\delta_{\rho}^{\mu} T_{\alpha}^{\alpha \nu}-\delta_{\rho}^{\nu} T_{\alpha}^{\alpha \mu}\right)$.

Applying $f(T)$ gravity in a cosmological framework, namely imposing the homogeneous and isotropic geometry $e_{\mu}^{A}=\operatorname{diag}(1, a, a, a)$, which corresponds to the spatially flat Friedmann-Robertson-Walker (FRW) metric

$$
d s^{2}=d t^{2}-a^{2}(t) \delta_{i j} d x^{i} d x^{j},
$$

with $a(t)$ the scale factor, and inserting it into the general field equations (4), we extract the Friedmann equations

$$
\begin{aligned}
& H^{2}=\frac{8 \pi G}{3} \rho_{m}-\frac{f}{6}+\frac{T f_{T}}{3} \\
& \dot{H}=-\frac{4 \pi G\left(\rho_{m}+p_{m}\right)}{1+f_{T}+2 T f_{T T}} .
\end{aligned}
$$

In the above equations $H \equiv \dot{a} / a$ is the Hubble function, with dots denoting derivatives with respect to $t$, and $\rho_{m}$, $p_{m}$ are respectively the energy density and pressure for the matter perfect fluid.

Let us now study the perturbations of $f(T)$ around an FRW cosmological background, focusing on the gravitational wave part. We follow [57] and we perturb the tetrads as

$$
e_{\mu}^{A}(x)=\bar{e}_{\mu}^{A}(x)+\chi_{\mu}^{A}(x)
$$

where $\bar{e}_{\mu}^{A}$ represents the part of the tetrad corresponding to metric components, which satisfies the equation $g_{\mu \nu}(x)=\eta_{A B} e_{\mu}^{A} e_{\nu}^{B}=\eta_{A B} \bar{e}_{\mu}^{A} \bar{e}_{\nu}^{B}$ (the part $\chi_{\mu}^{A}$ that represents the degrees of freedom released from the local Lorentz transformation is not going to play any role in the analysis of this work). The part $\bar{e}_{\mu}^{A}$ around a spatially flat FRW geometry writes as [21, 57, 62.

$$
\begin{aligned}
& \bar{e}_{\mu}^{0}=\delta_{\mu}^{0}(1+\psi)+a \delta_{\mu}^{i}\left(G_{i}+\partial_{i} F\right), \\
& \bar{e}_{\mu}^{a}=a\left[\delta_{\mu}^{a}(1-\phi)\right. \\
& \left.+\delta_{\mu}^{i} \delta^{a j}\left(\frac{1}{2} h_{i j}+\partial_{i} \partial_{j} B+\partial_{j} C_{i}+\partial_{i} C_{j}\right)\right], \\
& \bar{e}_{0}^{\mu}=\delta_{0}^{\mu}(1-\psi)-\frac{1}{a} \delta^{\mu i}\left(G_{i}+\partial_{i} F\right), \\
& \bar{e}_{a}^{\mu}=\frac{1}{a}\left[\delta_{a}^{\mu}(1+\phi)\right. \\
& \left.-\delta^{\mu i} \delta_{a}^{j}\left(\frac{1}{2} h_{i j}+\partial_{i} \partial_{j} B+\partial_{i} C_{j}+\partial_{j} C_{i}\right)\right],
\end{aligned}
$$

where $\phi, \psi, B$ and $F$ are the scalar modes, $C_{i}$ and $G_{i}$ the transverse vector modes, and $h_{i j}$ the transverse traceless 
tensor mode, which lead to the standard perturbed metric

$$
\begin{aligned}
g_{00} & =-1-2 \psi, \\
g_{i 0} & =-a\left[\partial_{i} F+G_{i}\right], \\
g_{i j} & =a^{2}\left[(1-2 \phi) \delta_{i j}+h_{i j}+\partial_{i} \partial_{j} B+\partial_{j} C_{i}+\partial_{i} C_{j}\right] .
\end{aligned}
$$

In the rest of the manuscript we set the scalar and vector perturbations to zero, since we are interested in studying the gravitational-wave sector.

Inserting (9) into (1) we acquire

$$
\begin{aligned}
T^{i}{ }_{0 j} & =H \delta_{i j}+\frac{1}{2} \dot{h}_{i j} \\
T^{i}{ }_{j k} & =\frac{1}{2}\left(\partial_{j} h_{i k}-\partial_{k} h_{i j}\right),
\end{aligned}
$$

and consequently the torsion scalar 2 is perturbed as

$$
T=T^{(0)}+O\left(h^{2}\right)=6 H^{2}+O\left(h^{2}\right),
$$

with $T^{(0)}$ the zeroth-order quantity. Thus, we deduce that at linear order the torsion scalar remains unaffected, which lies behind the fact that in $f(T)$ gravity the gravitational waves do not have extra polarization modes [59]. Moreover, the perturbed super-potential can be written as $S_{i}{ }^{0 j}=H \delta_{i j}-\frac{1}{4} \dot{h}_{i j}$ and $S_{i}{ }^{j k}=\frac{1}{4 a^{2}}\left(\partial_{j} h_{i k}-\partial_{k} h_{i j}\right)$. Inserting the perturbed quantities into the field equations (4), and neglecting the matter sector, we obtain [57.

$$
\begin{aligned}
& 4 f_{T}\left[\left(\dot{H}+3 H^{2}\right) \delta_{i j}+\frac{1}{4}\left(-\ddot{h}_{i j}+\frac{\nabla^{2}}{a^{2}} h_{i j}-3 H \dot{h}_{i j}\right)\right] \\
& +4 \dot{f}_{T}\left(H \delta_{i j}-\frac{\dot{h}_{i j}}{4}\right)-f \delta_{i j}=0
\end{aligned}
$$

where the derivative $f_{T}$ is calculated at $T=T^{(0)}$. Hence, the perturbation part of the above equation leads to the equation of motion for the gravitational waves in $f(T)$ cosmology, namely

$$
h_{i j}^{\prime \prime}+2 \mathcal{H}\left(1-\beta_{T}\right) h_{i j}^{\prime}+k^{2} h_{i j}=0,
$$

with primes denoting derivative with respect to the conformal time, and where we have introduced the dimensionless parameter [57]

$$
\beta_{T}=-\frac{\dot{f_{T}}}{3 H f_{T}} .
$$

Therefore, we straightforwardly deduce that the speed of GWs is equal to one, i.e. equal to the speed of light, and thus the experimental constraint of GW170817 is trivially satisfied in $f(T)$ gravity. However, as it was mentioned in [57, the correction term $\beta_{T}$ reflects the effect on the gravitational waves due to the change that $f(T)$ gravity brings on the background they propagate on.

In order to quantify the above effect we follow the analysis of 13,63 . In most of gravitational modifications one can bring the GW propagation equation in the form

$$
h_{i j}^{\prime \prime}+(2+\nu) \mathcal{H} h_{i j}^{\prime}+\left(c_{T}^{2} k^{2}+a^{2} \mu^{2}\right) h_{i j}=\Pi_{i j},
$$

where $c_{T}$ is the GW propagation speed, $\mu$ is the effective graviton mass, $\nu$ is related to the effectively running Planck mass, and $\Pi_{i j}$ is a source term arising from possible additional fields. Hence, one can describe the deviation of GW propagation at cosmological scales comparing to general relativity through

$$
h=e^{-\mathcal{D}} e^{-i k \Delta T} h_{G R},
$$

where

$$
\mathcal{D}=\frac{1}{2} \int \nu \mathcal{H} d \tau^{\prime}
$$

quantifies the amplitude modification, i.e. the damping factor, while

$$
\Delta T=\int\left(1-c_{T}-\frac{a^{2} \mu^{2}}{2 k^{2}}\right) d \tau^{\prime}
$$

quantifies the phase modification, i.e. the time delay.

In the case of $f(T)$ gravity Eq. (14) implies that $c_{T}=1, \mu=0, \Pi_{i j}=0$ and $\nu=-2 \beta_{T}$. Therefore, we deduce that in $f(T)$ gravity $\Delta T=0$, and thus we do not obtain any phase modification in comparison to general relativity. However, the quantity $\nu$, which carries the information of the $f(T)$ modification, will lead to a non-zero $\mathcal{D}$, and thus to an amplitude modification of the gravitational waves comparing to general relativity. Hence, this modification can in principle be measured in GW observations, offering an observational signature of this class of modified gravity. In the following sections we quantify this behavior.

\section{PRIMORDIAL GRAVITATIONAL WAVES}

In this section we investigate the deviation of $\mathrm{GW}$ propagation in $f(T)$ gravity, comparing to general relativity, focusing on the primordial gravitational waves. As a specific model, and without loss of generality, we will consider the power-law one, which is the most viable one, nevertheless our analysis can be performed for every $f(T)$ form. The power-law scenario corresponds to [19:

$$
f(T)=T+\alpha(-T)^{b} .
$$

with $\alpha$ and $b$ the two parameters. Inserting this into (6) at present time we obtain

$$
\alpha=\left(6 H_{0}^{2}\right)^{1-b}\left(\frac{1-\Omega_{m 0}-\Omega_{r 0}}{2 b-1}\right),
$$

with $\Omega_{m 0}, \Omega_{r 0}$ respectively the current values of the matter and radiation density parameters, and $H_{0}$ the Hubble parameter at present, and thus the only free model parameter is $b$. The value $b=0$ corresponds to recovery of general relativity and of $\Lambda \mathrm{CDM}$ cosmology (in this case the parameter $\alpha$ is related to the cosmological constant).

We insert the above power-law $f(T)$ form into the modified propagation equations of the previous section, 


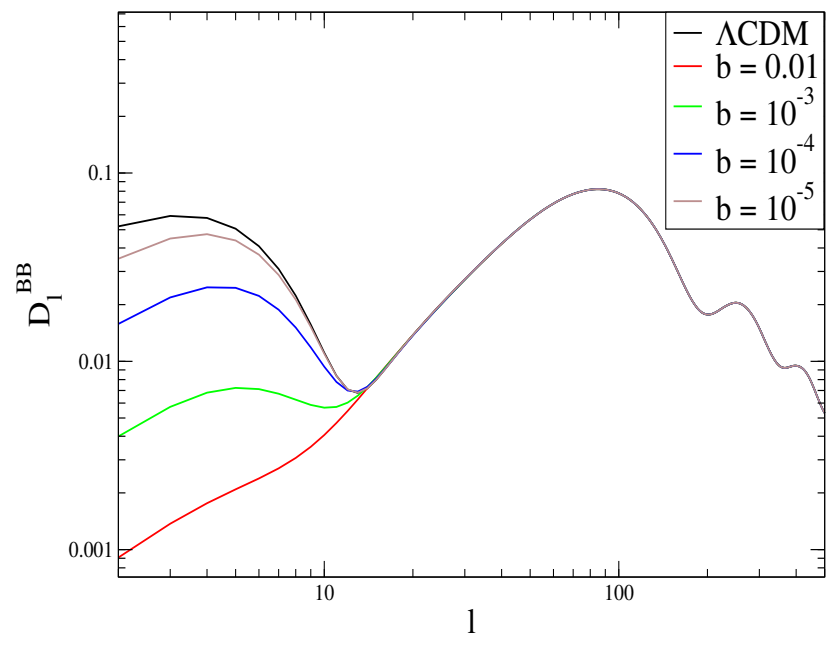

FIG. 1: The $C M B B B$ power spectrum, $D_{l}^{B B}=l(l+$ 1) $C_{l}^{B B} / 2 \pi \mu K$, for the $\Lambda C D M$ cosmology and the $f(T)$ gravity power-law model (20), for various values of the free model parameter $b$.

and we use the publicly available CLASS (Cosmic Linear Anisotropy Solving System) code 64 in order to investigate the effects of the gravitational waves on the $\mathrm{CMB}$ $\mathrm{BB}$ anisotropy and also to calculate the propagation of the gravitational waves through cosmic time.

In Fig. 1 we show the CMB BB spectrum for the $f(T)$ gravity and the $\Lambda \mathrm{CDM}$ cosmological model as the reference model, for tensor modes only. In drawing the graphs we have considered various values of the free model parameter $b$. All other parameters are fixed in the same way for both models, based on Planck 2015 results 65. As we observe, for the angular scale $l>20$, the theoretical predictions for $f(T)$ gravity and $\Lambda \mathrm{CDM}$ are practically identical. Thus, for small angular scales no deviations are expected compared to standard $\Lambda \mathrm{CDM}$ cosmology. On the other hand, we can see significant deviations at large angular scales.

Additionally, the theoretical CMB BB spectrum should also present a peak at $l \simeq 5$, still to be detected by future experiments, due to the effects of tensor modes on the scattering during the reionization epoch. At low- $l$, the large angular is dominated by modes that have not entered the horizon at recombination and therefore are approximately constant. Thus, the effects due to $f(T)$ modification can be quantified on the reionization peak (which should be located at $l \simeq 5$ ), where we can note different predictions for a range of values of model parameter $b$ compared to the reference $\Lambda \mathrm{CDM}$ scenario. Interestingly, future measurements of the reionization peak by CMB spectrum could be used to discriminate between $f(T)$ gravity and standard cosmology scenario.

In Fig. 22 following the approach of 63, we depict the propagation of GWs as the function of the scale factor, for various values of the model parameter $b$ of the powerlaw form (20), in order to investigate how $b$ affects the amplitude damping. It is already known that the ampli-
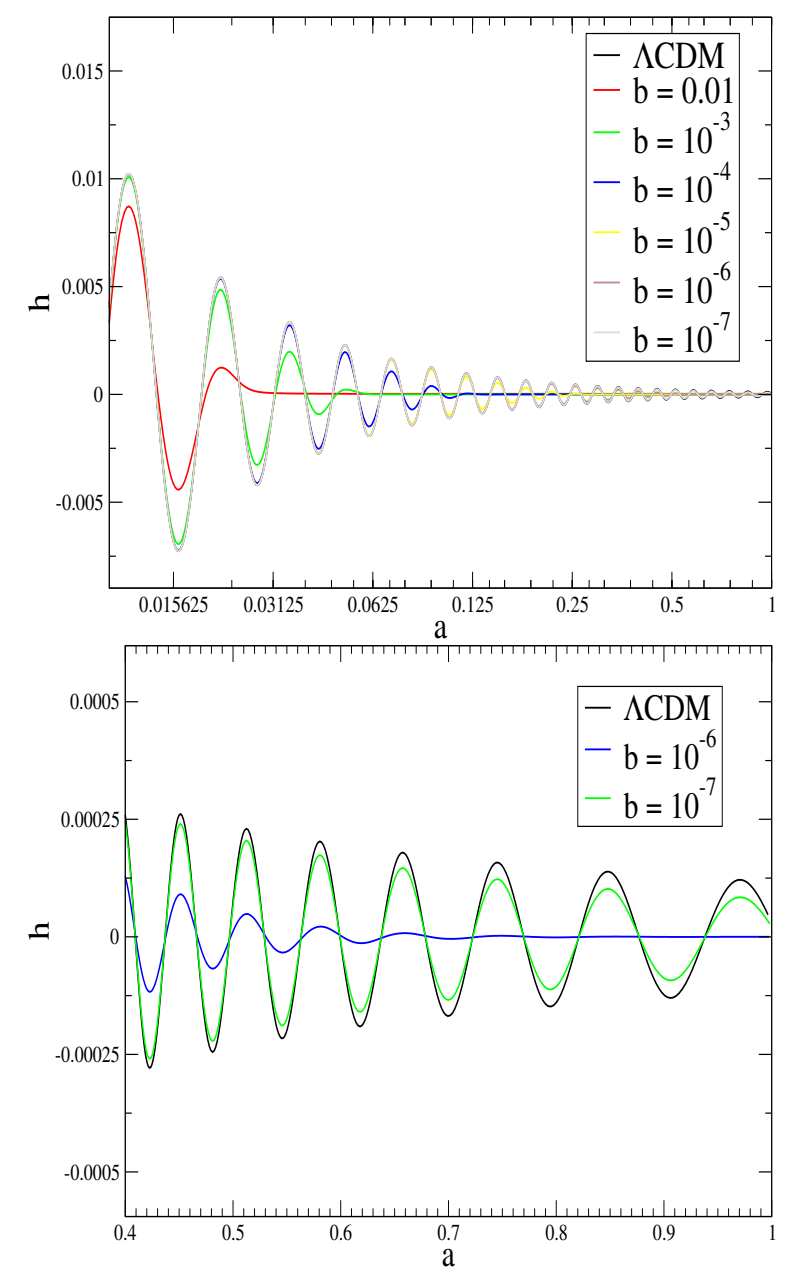

FIG. 2: Propagation of the gravitational waves at $k=$ $0.01 \mathrm{Mpc}^{-1}$, in $\Lambda C D M$ scenario and in the $f(T)$ gravity powerlaw model (20) for various values of the free model parameter $b$, as a function of the scale factor $a=(1+z)^{-1}$. Upper graph: full cosmological history. Lower graph: late-time cosmological history.

tude of GWs decays rapidly immediately after the tensormodes entry into the horizon, while before the entrance to the horizon the amplitude is practically constant. The expansion of the Universe leads to damping of the tensor modes with the term proportional to $h^{\prime}$. We have noted that for $a<0.01$, namely at early times, taking different ranges in orders of magnitude of $b$, only a tiny deviation from $\Lambda \mathrm{CDM}$ paradigm appears. On the other hand, for $a>0.01$ the damping factor becomes significant, and we can see that already at the matter era the amplitude of GWs becomes practically null (or extremely small) for $b>10^{-5}$. For instance, for $b=10^{-2}$ we have $h \simeq 0$ at $a \simeq 0.04$, while for $b=10^{-5}$ we get that $h \simeq 0$ at $a \simeq 0.4$. Moreover, when the Universe enters into the accelerated phase only for the cases $b=10^{-6}, 10^{-7}$ the tensor-modes oscillations are non-null, however in the case $b=10^{-6}$ the modes quickly decay and only in the case $b=10^{-7}$ they survive up to present time. Concerning the phase, we do 
not observe any difference, since as we discussed there is no phase modification in $f(T)$ gravity $(\Delta T=0$ in $(17))$.

In summary, the more $b$ departs from its general relativity value $b=0$ the larger is the GW amplitude decay comparing to $\Lambda$ CDM scenario. This is because for larger $b$ values the tensorial modes enter in the cosmological horizon earlier, compared to the standard prediction within $\Lambda$ CDM cosmology, and thus the GW amplitude goes rapidly to zero already in radiation and matter eras. Hence, we deduce that a possible future detection of primordial gravitational waves would imply that $b \lesssim 10^{-7}$ (taking the specific scale $k=0.01 \mathrm{Mpc}^{-1}$ ), bringing the viable $f(T)$ gravity models five orders of magnitude closer to $\Lambda \mathrm{CDM}$ cosmology comparing to standard cosmological constraints based on SN Ia, BAO, CMB, $H(z)$ data 51 56]. This fact reveals the capabilities of gravitationalwave astronomy, since such strong constraints were possible to be obtained only through Solar-System data 66].

\section{PLANCK AND CORE CONSTRAINTS}

In this section we present new observational constraints on $f(T)$ gravity, arising from the CMB data from Planck collaboration [65], as well as from forecasts that are expected from near-future probes such as CORE 67, 68. Similarly to the previous section we will use the CLASS code 64. We mention that in the present analysis we only consider the CMB data, neglecting the influences of the external data, since we are particularly interested in quantifying and analyzing the effects of equations (14)-(15), i.e. the modified GWs propagation in $f(T)$ gravity, as well as its effects on CMB. The $f(T)$ gravity has been recently well constrained from the geometrical data [53, 56, and using the CMB data for the first time in 55].

Concerning the Planck data we use the likelihood fake planck realistic included in MontePython code 69, taking into account the temperature, polarization and CMB lensing extraction, and we adopt noise spectra roughly matching those expected from the full Planck results. The baseline parameter space is given by

$$
\mathcal{P} \equiv\left\{100 \omega_{\mathrm{b}}, \omega_{\mathrm{cdm}}, \ln 10^{10} A_{s}, n_{s}, \tau_{\text {reio }}, H_{0}, r, b\right\},
$$

where the parameters in $\mathcal{P}$ from left to right are respectively the baryon density, the cold dark matter density, the amplitude and slope of the primordial spectrum of metric fluctuations, the scalar spectral index, the optical depth to reionization, the Hubble constant, the tensorto-scalar ratio, and the free parameter (i.e. $b$ ) of the power-law $f(T)$ model 200 . The priors used for the model parameters are the following: $\omega_{\mathrm{b}} \in[0.005,0.1]$, $\omega_{\mathrm{cdm}} \in[0.01,0.99], \ln 10^{10} A_{s} \in[2.4,4.0], n_{s} \in[0.5,1.5]$, $\tau_{\text {reio }} \in[0.01,0.8], H_{0} \in[50,90], r \in[0,1.0]$, and $b \in$ $[0,0.1]$.

Concerning the CORE analysis we use the likelihood CORE $m 5$ also included in MontePython. The experi- mental specifications for CORE data in our analysis are summarized in Table 【.

\begin{tabular}{c|c|c|c}
\hline Channel $[\mathrm{GHz}]$ & FWMH $[\operatorname{arcmin}]$ & $\Delta T$ & $\Delta P$ \\
\hline 130 & 8.51 & 3.9 & 5.5 \\
145 & 7.68 & 3.6 & 5.1 \\
160 & 7.01 & 3.7 & 5.2 \\
175 & 6.45 & 3.6 & 5.1 \\
195 & 5.84 & 3.5 & 4.9 \\
220 & 5.23 & 3.8 & 5.4 \\
\hline
\end{tabular}

TABLE I: Experimental specifications for CORE, with frequency channels dedicated to cosmology, and beam width, temperature sensitivity, and polarization sensitivity for each channel, in units of $\mu \mathrm{K}$ arcmin.

In our forecasts, we assume $l_{\min }=2, l_{\max }=3000$, and $f_{\text {sky }}=0.70$. We forecast the $f(T)$ gravity with the set of cosmological parameters shown in $\mathcal{P}$.

In the forecasting analysis we assume the fiducial values of the above parameters as: $\{2.22,0.119,3.07,0.962$, $0.05,68.0,0.1,0.005\}$. The details of the methodology used in the CORE likelihood can be seen in 67, 68.

In the upper graph of Fig. 3 we present the parametric space in the $n_{s}-r$ plane, where for the analysis we have assumed the pivot scale at $k=0.05 \mathrm{Mpc}^{-1}$, which is the standard in the literature. As we observe, $r<0.23$ at $95 \%$ confidence level (CL) and $n_{s}=0.96 \pm 0.005$ at $68 \%$ CL from the Planck data. These results are also in accordance with the Planck team assuming $\Lambda \mathrm{CDM}$ [70, where no evidence for a non-null $r$ is also reported and $n_{s}<1$ up to $5 \sigma \mathrm{CL}$. According to the authors' knowledge, this is the first observational constraints on inflation parameters (plane $n_{s}-r$ ) obtained directly in the context of $f(T)$ gravity.

In the lower graph of Fig. 3 we present the parametric space in the $H_{0}-b$ plane. As we can see, $H_{0}=69 \pm 5.42$ $\mathrm{km} / \mathrm{s} / \mathrm{Mpc}$ and $b=0.005 \pm 0.006$, both at $68 \%$ CL. As already noted in [55] (within a different theoretical and observational perspective), $f(T)$ gravity can provide high $H_{0}$ values, and therefore a possible solution to the $H_{0}$ tension might be available in this context [55].

In this work, incorporating the propagation modification of the tensor modes due to $f(T)$ gravity and its effects on $\mathrm{CMB}$, we can again see that $H_{0}$ is fully compatible with local measurements, namely $H_{0}=73.2 \pm 1.74$ [71, even at $1 \sigma$ CL.

We proceed motivated by the future CMB experiments, and we apply the inherent modifications due to $f(T)$ gravity aiming to impose bounds on the baseline parameters of the model. In the upper panel of Fig. 3 we additionally present the parametric space in the plane $n_{s}-r$ via CMB CORE forecasts. In this way we can see how much can CORE improve the constraints compared to the current Planck ones. We find $r=0.1 \pm 0.020$ and $n_{s}=0.96 \pm 0.0017$ at $68 \%$ CL (as we mentioned above, we assume $r=0.1$ and $n_{s}=0.96$ in the forecast 

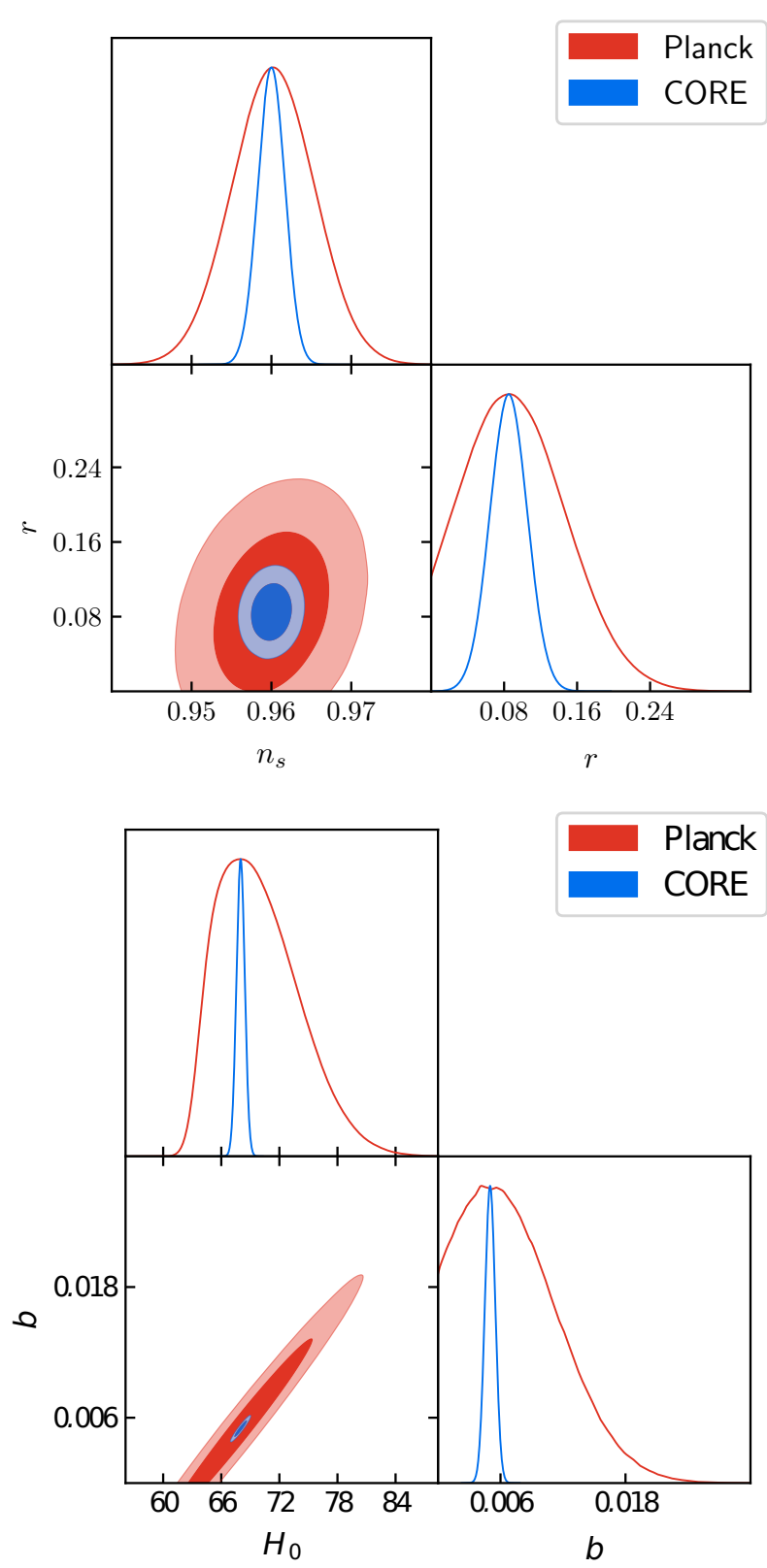

FIG. 3: Upper graph: One-dimensional marginalized posterior distributions and $68 \%, 95 \% C L$ regions in the $n_{s}-r$ plane at $k=0.05 \mathrm{Mpc}^{-1}$, from the Planck data and CORE forecasts. Lower graph: One-dimensional marginalized posterior distributions and $68 \%, 95 \%$ CL regions in the $H_{0}-n$ plane (where $b$ is the free parameter of the $f(T)$ gravity model (20)) from the Planck data and CORE forecasts.

data simulation). Let us define the improvement with respect to the constraints arising from the Planck data as $I_{i}=\sigma_{\text {Planck }} / \sigma_{C O R E}{ }^{1}$. Thus, for the parameters of our interest, we find that $I_{r}=11.5$ and $I_{n_{s}}=2.94$. Within

\footnotetext{
${ }^{1}$ The label $i$ runs over the parameters given in $\mathcal{P}$.
}

the $\Lambda$ CDM paradigm, the CORE collaboration 68] finds for instance $r=0.0042 \pm 0.00028$, assuming $r=0.0042$ in the forecast analysis. For other forecastings using CORE estimation see 67, 68.

In the lower graph of Fig. 3 we depict the bounds that CORE results can impose directly on the free model parameter of the power-law $f(T)$ model, and in particular we find that $b=0.0050 \pm 0.00049$. Here, we note an improvement of $I_{b}=12.2$. As we can see, future constraints could improve current constraints with respect to the Planck data, on the $f(T)$ gravity free parameter, by a factor of 12. A precise measurement of this parameter, with that magnitude of improvement, can offer an opportunity to check deviations from the general theory of relativity. The value $b=0.0050$ is the best-fit value that arises from the Planck data too, thus it is reasonable to consider it for performing our forecast simulation. Hence, we may conclude that the forecast errors on $b$ may limit a non-null value on this parameter by taking future CMB missions. This will be a clear signature that a deviation from $\Lambda \mathrm{CDM}$ cosmology is favored.

\section{GRAVITATIONAL WAVES FROM MERGERS}

In the previous section we investigated the effects of $f(T)$ gravity on the primordial GWs. In this section we present a preliminary discussion on the possibility to use the properties of the detected GWs that arise from mergers, in order to impose constraints on $f(T)$ gravity and on modified gravity in general. In this direction, the GWs that are accompanied by electromagnetic counterparts are proved to be extremely efficient.

In the case of detection of GWs that arise from black holes mergers ones does not have any other information apart from the properties of the GWs at the moment they reach earth, and the direction in space they came from (in the case where three different detectors are used). Hence, assuming that general relativity is the underlying gravitational theory and that $\Lambda \mathrm{CDM}$ cosmology is the background cosmological model one can calculate the distance of the merger, the involved black hole masses, and the properties of the GWs at the moment of emission [16]. Definitely, assuming a modified gravity and its implied cosmology as the underlying theory will lead to different calculations, and thus one faces degeneracies that do not allow for distinguishing different theories.

However, the situation changes radically in the case of detection of GWs that arise from neutron stars mergers, which are accompanied by an electromagnetic counterpart. In this case apart from the properties of the GWs at the moment they reach earth and their origin direction, one can additionally calculate their propagation speed, the distance of the merger, and the involved neutron stars masses, independently of the underlying gravitational theory and cosmological scenario, just using the implied physics from the electromagnetic infor- 
mation [7, 8]. Therefore, without assuming an underlying cosmology one can find the properties of the GWs at the time of their emission too. Hence, knowing the GWs properties at both the emission and detection time one can extract information for the background on which they propagated and thus impose constraints on the gravitational theory that determines it. In the case of $f(T)$ gravity, as we described in Section II] the effect of the $f(T)$ modification on the underlying cosmology leads to an amplitude modification comparing to the GWs propagating in $\Lambda \mathrm{CDM}$ scenario under general relativity, without a phase change.

In principle one can follow the above roadmap and impose new constraints on $f(T)$ gravity. In practice however, the involved procedure is very complicated and one needs to perform a thorough investigation on the GWs generation from neutron star mergers in the framework of $f(T)$ gravity itself. In these lines the analysis can be based on the recent work [14, focusing on the strain amplitude of the generated Gws. Since the necessary calculations are lengthy and complicated and lie beyond the scope of the present work, which is mainly the investigation of cosmological GWs, it is left for a separate project.

\section{CONCLUSIONS}

The advancing GWs multi-messenger astronomy offers a new window to observe nature, and amongst others to extract information about cosmology and gravity. In this work we focused on the case of $f(T)$ gravity, and we studied the new observational constraints that arise from the effect of primordial GWs on the cosmic microwave background (CMB) anisotropies and the BB spectrum.

The main feature of the investigation that lies at the basis of the analysis, is that the underlying gravitational theory determines both the properties of the GWs themselves (speed, polarization modes, etc) as well as the properties of the background on which they propagate (the expanding universe). Hence, one can use the GW features in order to impose constraints on the various gravitational theories and offer a way to distinguish them. In the particular case of $f(T)$ gravity (where it was recently shown that GWs propagate with the light speed [57, 58, without extra polarization modes [35), we showed that one obtains only an amplitude modification and not a phase one on the GWs propagation, comparing to the case of general relativity in the background of $\Lambda$ CDM cosmology.

Concerning primordial GWs, and focusing without loss of generality on the power-law $f(T)$ model, we showed that the more the model departs from general relativity, i.e. the more the exponent $b$ departs from its general relativity value $b=0$, the larger is the GW amplitude decay comparing to $\Lambda$ CDM scenario. Hence, we deduced that a possible future detection of primordial gravitational waves would imply that $b \lesssim 10^{-7}$ (analyzing on the scale $k=0.01 \mathrm{Mpc}^{-1}$ ), bringing the viable $f(T)$ gravity models five orders of magnitude closer to $\Lambda$ CDM cosmology comparing to standard cosmological constraints based on SN Ia, BAO, CMB, $H(z)$ data [51 56]. This fact reveals the capabilities of gravitational-wave astronomy, since such strong constraints were possible to be obtained only through Solar-System data [66].

Additionally, we used the CLASS code in order to quantify the primordial GWs effect on the CMB anisotropies and the BB spectrum. We used both the data from the Planck probe, as well as forecasts from the near-future CORE collaboration. As we showed, possible non-trivial constraints on the tensor-to-scalar ratio would favor a model parameter $b$ different from its general relativity value, offering a clear signature of $f(T)$ gravity comparing to $\Lambda \mathrm{CDM}$ cosmology.

Finally, we examined the possibility of constraining $f(T)$ gravity through the detection of GWs that arise from neutron stars mergers, which are accompanied by an electromagnetic counterpart. In this case, apart from observing the properties of the GWs at the time of detection one can use the features of the electromagnetic observations and the deduced physics of the neutron stars to find the properties of the GWs at the time of their emission, without any assumption on the underlying theory of gravity and the background cosmological evolution. Hence, knowing the GWs properties at both the emission and detection time it is possible to extract information for the background on which they propagated and thus impose constraints on the gravitational theory that determines it. Since the basic scope of the present work is mainly the investigation of cosmological Gws, the above detailed investigation is left for a separate project.

In summary, we showed how one can use information from the advancing multi-messenger GW astronomy in order to extract new observational constraints on $f(T)$ gravity. As we saw, $f(T)$ gravity remains in agreement with observations and thus a good candidate for the description of nature.

\section{Acknowledgments}

The authors thank the referee for some specific clarifying points. The authors would also like to thank J. C. N. de Araujo and M. E. S. Alves for useful discussions. This article is based upon work from CANTATA COST (European Cooperation in Science and Technology) action CA15117, EU Framework Programme Horizon 2020.
[1] B. P. Abbott et al. [LIGO Scientific and Virgo Collaborations], Phys. Rev. Lett. 116, 061102 (2016).
[2] B. P. Abbott et al. [LIGO Scientific and Virgo Collabo- 
rations], Phys. Rev. Lett. 116, 241103 (2016).

[3] B. P. Abbott et al. [LIGO Scientific and VIRGO Collaborations], Phys. Rev. Lett. 118, 221101 (2017).

[4] B . P. .Abbott et al. [LIGO Scientific and Virgo Collaborations], Astrophys. J. 851, L35 (2017).

[5] B. P. Abbott et al. [LIGO Scientific and Virgo Collaborations], Phys. Rev. Lett. 119, 141101 (2017).

[6] B. P. Abbott et al. [LIGO Scientific and Virgo Collaborations], Astrophys. J. 832, L21 (2016).

[7] B. P. Abbott et al. [LIGO Scientific and Virgo Collaborations], Phys. Rev. Lett. 119, 161101 (2017).

[8] B. P. Abbott et al. [LIGO Scientific and Virgo Collaborations], The Astrophysical Journal Letters 848, L13 (2017).

[9] Y. Akrami et al., [Planck Collaboration], arXiv:1807.06211 [astro-ph.CO].

[10] T. Baker, E. Bellini, P. G. Ferreira, M. Lagos, J. Noller, and I. Sawicki, Phys. Rev. Lett. 119, 251301 (2017).

[11] P. Creminelli and F. Vernizzi, Phys. Rev. Lett. 119, 251302 (2017).

[12] J. Sakstein and J. Jain, Phys. Rev. Lett. 119, 251303 (2017)

[13] J. M. Ezquiaga and M. Zumalacrregui, Phys. Rev. Lett. 119, no. 25, 251304 (2017).

[14] J. M. Ezquiaga and M. Zumalacrregui, arXiv:1807.09241 [astro-ph.CO].

[15] L. Lombriser and A. Taylor, JCAP 03031 (2016).

[16] L. Lombriser and N. A. Lima, Phys. Lett. B 765, 382 (2017).

[17] Y. F. Cai, S. Capozziello, M. De Laurentis and E. N. Saridakis, Rept. Prog. Phys. 79, no. 10, 106901 (2016).

[18] R. Ferraro and F. Fiorini, Phys. Rev. D 75, 084031 (2007).

[19] G. R. Bengochea and R. Ferraro, Phys. Rev. D 79, 124019 (2009).

[20] E. V. Linder, Phys. Rev. D 81, 127301 (2010).

[21] S. H. Chen, J. B. Dent, S. Dutta and E. N. Saridakis, Phys. Rev. D 83, 023508 (2011).

[22] J. B. Dent, S. Dutta and E. N. Saridakis, JCAP 1101, 009 (2011).

[23] R. Zheng and Q. G. Huang, JCAP 1103, 002 (2011).

[24] Y. Zhang, H. Li, Y. Gong and Z. H. Zhu, JCAP 1107, 015 (2011).

[25] Y. -F. Cai, S. -H. Chen, J. B. Dent, S. Dutta, E. N. Saridakis, Class. Quant. Grav. 28, 215011 (2011).

[26] S. Capozziello, V. F. Cardone, H. Farajollahi and A. Ravanpak, Phys. Rev. D 84, 043527 (2011).

[27] C. Q. Geng, C. C. Lee, E. N. Saridakis and Y. P. Wu, Phys. Lett. B 704, 384 (2011).

[28] Y. P. Wu and C. Q. Geng, Phys. Rev. D 86, 104058 (2012).

[29] J. de Haro and J. Amorós, Phys. Rev. Lett. 110, no. 7, 071104 (2013).

[30] W. D. Guo, Q. M. Fu, Y. P. Zhang and Y. X. Liu, Phys. Rev. D 93, no. 4, 044002 (2016).

[31] K. Atazadeh and F. Darabi, Eur.Phys.J. C72 (2012) 2016.

[32] S. Basilakos, S. Capozziello, M. De Laurentis, A. Paliathanasis and M. Tsamparlis, Phys. Rev. D 88, 103526 (2013).

[33] Y. C. Ong, K. Izumi, J. M. Nester and P. Chen, Phys. Rev. D 88 (2013) 2, 024019

[34] J. Amoros, J. de Haro and S. D. Odintsov, Phys. Rev. D 87, 104037 (2013).
[35] K. Bamba, S. Capozziello, M. De Laurentis, S. 'i. Nojiri and D. Sáez-Gómez, Phys. Lett. B 727, 194 (2013).

[36] A. Paliathanasis, S. Basilakos, E. N. Saridakis, S. Capozziello, K. Atazadeh, F. Darabi and M. Tsamparlis, Phys. Rev. D 89, 104042 (2014).

[37] G. Kofinas and E. N. Saridakis, Phys. Rev. D 90, 084045 (2014).

[38] C. Q. Geng, C. Lai, L. W. Luo and H. H. Tseng, Phys. Lett. B 737, 248 (2014).

[39] W. El Hanafy and G. G. L. Nashed, Eur. Phys. J. C 75, 279 (2015).

[40] S. Capozziello, O. Luongo and E. N. Saridakis, Phys. Rev. D 91, no. 12, 124037 (2015).

[41] M. Malekjani, N. Haidari and S. Basilakos, Mon. Not. Roy. Astron. Soc. 466, no. 3, 3488 (2017).

[42] G. Farrugia and J. L. Said, Phys. Rev. D 94, no. 12, 124054 (2016).

[43] N. Sk, Phys. Lett. B 775, 100 (2017).

[44] S. Bahamonde, C. G. Böhmer and M. M. Krššák, Phys. Lett. B 775, 37 (2017).

[45] M. Hohmann, L. Jrv and U. Ualikhanova, Phys. Rev. D 97, no. 10, 104011 (2018).

[46] S. Ilijic and M. Sossich, Phys. Rev. D 98, no. 6, 064047 (2018).

[47] A. Golovnev and T. Koivisto, arXiv:1808.05565 [gr-qc].

[48] A. I. Keskin, Eur. Phys. J. C 78, no. 9, 705 (2018).

[49] X. M. Deng, Class. Quant. Grav. 35, no. 17, 175013 (2018).

[50] A. El-Zant, W. El Hanafy and S. Elgammal, arXiv:1809.09390 [gr-qc].

[51] P. Wu and H. W. Yu, Phys. Lett. B 693, 415 (2010).

[52] S. Nesseris, S. Basilakos, E. N. Saridakis and L. Perivolaropoulos, Phys. Rev. D 88, 103010 (2013).

[53] R. C. Nunes, S. Pan and E. N. Saridakis, JCAP 1608, no. 08, 011 (2016).

[54] R. C. Nunes, A. Bonilla, S. Pan and E. N. Saridakis, Eur. Phys. J. C 77, no. 4, 230 (2017).

[55] R. C. Nunes, JCAP 1805, no. 05, 052 (2018).

[56] S. Basilakos, S. Nesseris, F. K. Anagnostopoulos and E. N. Saridakis, JCAP 1808, no. 08, 008 (2018).

[57] Y. F. Cai, C. Li, E. N. Saridakis and L. Xue, Phys. Rev. D 97, no. 10, 103513 (2018).

[58] C. Li, Y. Cai, Y. F. Cai and E. N. Saridakis, JCAP 1810, 001 (2018).

[59] G. Farrugia, J. L. Said, V. Gakis and E. N. Saridakis, Phys. Rev. D 97, no. 12, 124064 (2018).

[60] M. Hohmann, C. Pfeifer, J. L. Said and U. Ualikhanova, arXiv:1808.02894 [gr-qc].

[61] R. Aldrovandi, J.G. Pereira, Teleparallel Gravity: An Introduction, Springer, Dordrecht, 2013.

[62] Y. P. Wu and C. Q. Geng, JHEP 1211, 142 (2012).

[63] A. Nishizawa, Phys. Rev. D 97, 104037 (2018).

[64] D. Blas, J. Lesgourgues and T. Tram, JCAP 1107, 034 (2011).

[65] P. A. R. Ade et al. [Planck Collaboration], Astron. Astrophys. 594, A13 (2016).

[66] L. Iorio and E. N. Saridakis, Mon. Not. Roy. Astron. Soc. 427, 1555 (2012).

[67] E. Di Valentino et al. [CORE Collaboration], JCAP 1804, 017 (2018).

[68] F. Finelli et al. [CORE Collaboration], JCAP 1804, 016 (2018).

[69] B. Audren, J. Lesgourgues, K. Benabed and S. Prunet, JCAP 1302, 001 (2013). 
[70] Y. Akrami et al., arXiv:1807.06211 [astro-ph.CO].

[71] A. G. Riess et al., Astrophys. J. 826, 56 (2016). 